\title{
Alterstice
}

Revue internationale de la recherche interculturelle

International Journal of Intercultural Research

Revista International de la Investigacion Intercultural

\section{Prendre en compte la diversité à l'école en se rappelant sa complexité comme institution}

\section{Fasal Kanouté}

Volume 6, numéro 1, 2016

Prendre en compte la diversité à l'école

URI : https://id.erudit.org/iderudit/1038274ar

DOI : https://doi.org/10.7202/1038274ar

Aller au sommaire du numéro

Éditeur(s)

Alterstice

ISSN

1923-919X (numérique)

Découvrir la revue

Citer ce document

Kanouté, F. (2016). Prendre en compte la diversité à l'école en se rappelant sa complexité comme institution. Alterstice, 6(1), 9-12.

https://doi.org/10.7202/1038274ar
Résumé de l'article

La diversité en lien avec la pluriethnicité est l'objet de réflexions et de recherches sous différentes perspectives et à divers niveaux de fonctionnement de nos sociétés. La perspective interculturelle est souvent envisagée comme une réponse pour décrypter les enjeux des dynamiques d'acculturation. Elle permettrait aussi d'atténuer les effets du décalage entre les pratiques des milieux scolaires et les réalités socioculturelles d'élèves immigrants ou issus de minorités ethnoculturelles. L'objectif de ce numéro thématique est de s'inscrire dans la continuité des travaux dans ce domaine en actualisant la réflexion et en ayant l'ambition de l'ancrer dans les différentes déclinaisons institutionnelles de l'école. 


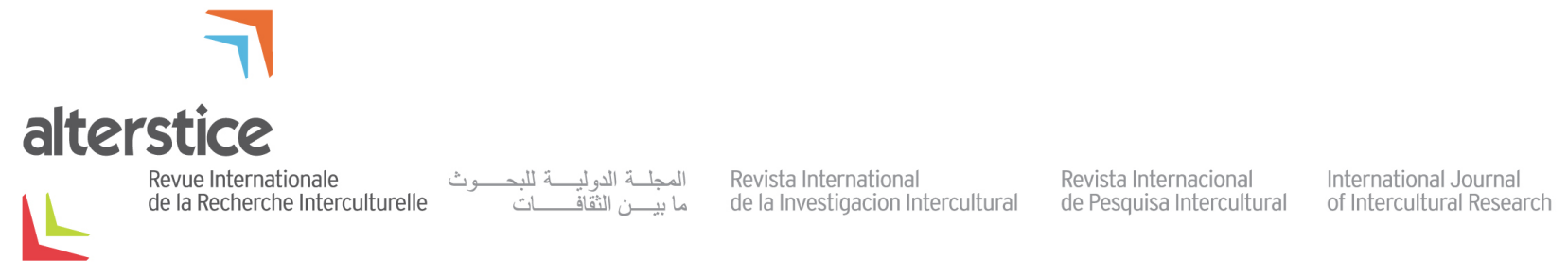

INTRODUCTION THÉMATIQUE

\section{Prendre en compte la diversité à l'école en se rappelant sa complexité comme institution}

Fasal Kanouté ${ }^{1}$

\section{Résumé}

La diversité en lien avec la pluriethnicité est l'objet de réflexions et de recherches sous différentes perspectives et à divers niveaux de fonctionnement de nos sociétés. La perspective interculturelle est souvent envisagée comme une réponse pour décrypter les enjeux des dynamiques d'acculturation. Elle permettrait aussi d'atténuer les effets du décalage entre les pratiques des milieux scolaires et les réalités socioculturelles d'élèves immigrants ou issus de minorités ethnoculturelles. L'objectif de ce numéro thématique est de s'inscrire dans la continuité des travaux dans ce domaine en actualisant la réflexion et en ayant l'ambition de l'ancrer dans les différentes déclinaisons institutionnelles de l'école.

\section{Rattachement de l'auteure}

${ }^{1}$ Université de Montréal, Montréal, Canada

\section{Correspondance}

fasal.kanoute@umontreal.ca

\section{Mots clés}

diversité, école, intégration, institution scolaire, perspective interculturelle.

\section{Pour citer cet article}

Kanouté, F. (2016). Prendre en compte la diversité à l'école en se rappelant sa complexité comme institution. Alterstice, 6(1), 9-12. 
À des degrés divers, nos sociétés contemporaines sont structurellement diversifiées, à la fois sur les plans socioculturel, linguistique et ethnique. Des débats sociétaux, souvent intenses, portant sur la prise en compte de cette diversité, ont tendance à se cristalliser sur l'enjeu de l'immigration, pour des raisons d'instrumentalisation politique à l'interne, pour des motifs relatifs à diverses conjonctures de la géopolitique mondiale, mais aussi parce que les institutions, dont l'école, font face à des impératifs de mobilisation pour résoudre des problèmes en lien avec l'accueil d'élèves et d'étudiants immigrants. II nous semble cependant important de ne pas considérer l'immigration récente comme l'unique facteur de création de la diversité de nos sociétés, même si les flux migratoires alimentent continuellement son hétérogénéité et actualisent les enjeux de la prise en compte de cette dernière.

La diversité en lien avec la pluriethnicité est l'objet de réflexions et de recherches sous différentes perspectives et à divers niveaux de fonctionnement de nos sociétés. La perspective interculturelle est souvent envisagée, au Québec notamment, comme une réponse pour décrypter les enjeux des dynamiques d'acculturation (Berry et Sabatier, 2010; Bouchard, 2012). Selon Clanet (1990), I'interculturalité se structure autour de processus (psychiques, relationnels, groupaux, institutionnels) générés par des interactions de cultures, dans un rapport d'échanges réciproques. Du point de vue de Lorcerie (2003, p. 272), cette perspective est sociale et politique : « II s'agit de contribuer à ce qu'advienne un "régime de tolérance" des différences moins marqué par le soupçon à l'égard des minoritaires, plus inclusif. C'est en somme prendre parti pour une décrispation de la culture sociale et politique [...] ». Pour White (2014, p. 37), I'interculturalisme relève "d'une orientation épistémologique qui se caractérise par le souci d'autrui dans une éthique relationnelle ». Parlant de "domaines contemporains de la diversité ", Vatz Laaroussi et Gélinas (2013) soutiennent que cette dernière se redéfinit constamment et qu'il faut sortir l'interculturel des sentiers battus. Cette réflexion sur la diversité résonne dans les enjeux scolaires.

La Politique sur l'intégration scolaire et l'éducation interculturelle du ministère de l'Éducation du Québec (MELS, 1998) définit l'interculturalisme autour de trois principes d'action: une égalité des chances de réussite, une maîtrise du français, langue commune de la vie publique et une éducation à la citoyenneté démocratique dans un contexte pluraliste. Elle l'envisage aussi comme « toute démarche éducative visant à faire prendre conscience de la diversité, particulièrement ethnoculturelle, qui caractérise le tissu social et à développer une compétence à communiquer avec des personnes aux référents divers, de même que des attitudes d'ouverture, de tolérance et de solidarité " (MEQ, 1998, p. 2). La perspective interculturelle permettrait aussi d'atténuer les effets du décalage entre les pratiques des milieux scolaires et les réalités socioculturelles d'élèves immigrants ou issus de minorités ethnoculturelles. Il est important de noter que ce décalage n'est pas seulement affaire de distance objective entre les caractéristiques des deux milieux, il est aussi construit par leur (non) rapport. Au Québec, au-delà de la volonté politique affichée, des chercheurs ont étudié de manière critique l'effectivité de la prise en compte de la diversité par l'école. Nous pensons particulièrement aux travaux de l'axe "Éducation » du Centre d'études ethniques des universités montréalaises. II ressort de ces travaux la nécessité d'imprimer un certain hétérocentrisme dans l'élaboration du cursus, dans l'analyse des enjeux éducatifs, dans l'organisation des services scolaires, dans la gestion du quotidien de l'école ainsi que dans la collaboration avec les familles et la communauté (Kanouté, 2007; Kanouté et Lafortune, 2014; Kanouté et Vatz Laaroussi, 2008).

L'objectif de ce numéro thématique, composé de dix articles, est de s'inscrire dans la continuité des travaux évoqués ci-dessus en actualisant la réflexion et en ayant l'ambition de l'ancrer dans les différentes déclinaisons institutionnelles de l'école. En effet, selon Bonny (2012), pour comprendre comment fonctionne et réagit une institution, il faut appréhender sa complexité à travers ses valeurs et principes, ses finalités, son cadre organisationnel, ses modes de régulation, les logiques professionnelles qui s'y déploient. Selon cet auteur, le politique définit l'institution, mais "le sens de l'institution s'élabore» aussi dans le quotidien, par l'action de différents acteurs qui doivent composer avec les tensions que génère la vie institutionnelle. Pour ce qui est de l'école, sa spécificité comme institution tient en partie aux protagonistes de la scolarisation: les élèves et étudiants, les enseignants, les autres professionnels, les gestionnaires, les parents d'élèves, les intervenants communautaires, etc. Neuf articles de ce numéro ont pour contexte le Canada (Québec, Ontario, ColombieBritannique, Alberta et Manitoba) et un article porte sur la situation en France (texte de Nyambek Kanga-Mebenga Nana). La situation de l'élève, de l'étudiant, de l'enfant et du jeune est au centre des enjeux discutés dans chaque article. Les quatre premiers textes articulent leur discours et des fragments observés de leurs vécus en classe ou en 
dehors de l'école. Les cinq suivants appréhendent cette situation du point de vue de différents protagonistes (enseignants, personnel de direction, parents). Les deux derniers abordent de manière explicite les enjeux et défis de l'élaboration des cursus en lien avec la prise en compte de la diversité. Ainsi, tout en reprenant des thématiques au cœur des perspectives de prise en compte de la diversité, sur fond explicite d'interculturalisme ou en creux, ce numéro révèle en même temps différentes parties du corps institutionnel qu'est l'école, et qui constituent autant de lieux d'intervention pour la faire bouger.

1. Dans le premier article (Kanouté, Gosselin-Gagné, Guennouni-Hassani et Girard), des élèves montréalais issus de l'immigration témoignent de facettes de leur identification ethnoculturelle et de la manière avec laquelle ils appréhendent l'altérité dans le réseau des pairs. Ils soulignent les défis rencontrés dans leur appropriation du programme, notamment l'acquisition de la langue d'enseignement, le français. Ils partagent quelques facteurs de résilience sociale et scolaire, notamment le recours aux ressources communautaires.

2. À travers l'analyse de répertoires linguistiques d'élèves immigrants du primaire à Montréal, le deuxième article (Lory et Armand) fait la preuve que lorsque l'école ignore l'altérité linguistique parmi les élèves, cela génère un impact sur les représentations que les élèves ont des langues qu'ils possèdent, et cet impact ne consolide pas leur capital linguistique. Les auteures de l'article soulignent la nécessité de valoriser la diversité linguistique et culturelle à l'école.

3. À travers l'expérience de jeunes de diverses origines ethnoculturelles dans des clubs de sciences après l'école, les auteurs du troisième article (Rahm, Malo et Lepage) décrivent les bénéfices que les jeunes retirent de ces activités ainsi que les défis rencontrés. La plus-value des activités parascolaires est soulignée, comme tuteur de résilience pour ces jeunes et comme outil d'intégration sociale des élèves immigrants. Les auteurs plaident pour une alliance communautaire autour du jeune, incluant l'école, les ressources de la communauté mais aussi les programmes de formation des enseignants à l'université.

4. Le quatrième article (Arcand, Asselin et Kanouté) décrit les défis qui jalonnent le processus d'acculturation d'étudiants récemment immigrés, en lien avec leur persévérance aux études, dans le contexte de l'Université de Montréal et de ses écoles affiliées. Les données révèlent des problèmes d'adaptation institutionnelle, des écueils dans l'intégration sociale et différentes barrières systémiques qui font écho à d'autres difficultés (vécues ou appréhendées) dans la société en général, notamment celles d'avoir un emploi à la hauteur de ses qualifications.

5. L'intégration d'élèves d'origine africaine en milieu scolaire francophone au Manitoba fait l'objet du cinquième article. Les auteurs (Atangana-Abé et $\mathrm{Ka}$ ) abordent la spécificité de l'acculturation de l'immigrant dans une communauté francophone, elle-même minoritaire, dans une province majoritairement anglophone. Du discours d'acteurs scolaires siégeant sur des comités de parents émergent des défis d'ordre économique, culturel et pédagogique, ainsi que des stratégies multidirectionnelles ciblant les enfants, les parents et les enseignants.

6. Dans le sixième article, les auteurs (Liboy et Mulatris) confrontent les perspectives d'enseignants albertains, non immigrants et d'origine immigrante, sur les défis de la relation entre l'école et les familles immigrantes. Pour créer une synergie dans l'action collective des acteurs scolaires en direction de ces familles, il y a lieu de cerner les convergences et les divergences entre ces perspectives, à propos de problématiques diverses allant des besoins linguistiques des familles à leurs attentes vis-à-vis de l'école.

7. S'appuyant sur le paradigme des "nouvelles mobilités ", l'auteure du septième article (Farmer) croise les regards d'élèves et d'acteurs scolaires (enseignants, directrice) sur le rapport de l'individu à ses différentes appartenances-allégeances, dans une école francophone en Ontario. S'inscrire dans un tel paradigme permet à l'école de s'adapter à la mobilité, de concevoir une fluidité des frontières, d'estomper l'opposition entre « ici » et " ailleurs », de casser l'aura d'étrangeté qui enveloppe l'Autre.

8. Avec en toile de fond le télescopage des représentations que les parents immigrants ont des systèmes scolaires de leur pays d'origine et du Québec, l'auteure (Charette) révèle dans le huitième article la complexité des stratégies mobilisées par ces parents dans le suivi scolaire de leurs enfants. Ces stratégies sont 
déployées à la maison, dans la communauté et à l'école. Plusieurs d'entre elles restent invisibles aux yeux de l'école, parce que méconnues ou non valorisées par cette dernière.

9. Le traitement des questions "socialement vives " par le programme de l'école en France est abordé dans le neuvième article. L'auteure (Nyambek Kanga-Mebenga Nana) recueille les points de vue de collégiens ( " minoritaires » et " majoritaires ») sur les socialisations concurrentielles dans les familles et l'appropriation des héritages historiques à l'école. Elle discute des défis relatifs à ces processus, de la construction d'un sentiment national et de l'élaboration d'un "socle commun de connaissances et de compétences ».

10. Comment un énoncé de politique balise-t-il la prise en compte de la diversité par l'institution scolaire? L'auteure du dixième article (Jacquet) fait une analyse critique du " nouveau » cadre éducatif en ColombieBritannique. Une tension dans cet énoncé réside dans « l'articulation de la dyade inclusion-diversité au travers du développement des compétences transversales ». L'auteure appelle à une vigilance dans la mise en œuvre des idées fortes de ce cadre.

\section{Références bibliographiques}

Berry, J. et Sabatier, C. (2010). Acculturation, discrimination, and adaptation among second generation immigrant youth in Montreal and Paris. International Journal of Intercultural Relations, 34(3), 191-207.

Bonny, Y. (2012). Les institutions publiques au prisme de la pluralité. Dans Y. Bonny et L. Demailly (dir.), L'institution plurielle (p. 9-36). Villeneuve d'Ascq (France) : Presses universitaires du Septentrion.

Bouchard, G. (2012). L'interculturalisme : un point de vue québécois. Montréal : Boréal.

Clanet, C. (1990). L'interculturel. Introduction aux approches interculturelles en éducation et en sciences humaines. Toulouse : Presses universitaires du Mirail.

Kanouté, F. (2007). La pratique de l'interculturel. Dans C. Solar et F. Kanouté (dir.), Équité en éducation et formation (p. 121-140). Montréal : Éditions Nouvelles.

Kanouté, F. et Lafortune, G. (2014). L'intégration des familles d'origine immigrante : les enjeux sociosanitaires et scolaires. Montréal : Presses de l’Université de Montréal.

Kanouté, F. et Vatz Laaroussi, M. (rédactrices invitées) (2008). Relations écoles - familles de minorités ethnoculturelles. Montréal : Revue des sciences de l'éducation (numéro thématique), 24(2).

Lorcerie, F. (2003). L'école et le défi ethnique. Paris : ESF.

Ministère de l'Éducation, du Loisir et du Sport (MELS). (1998). Une école d'avenir : politique d'intégration scolaire et d'éducation interculturelle. Québec : MELS.

Vatz Laaroussi, M. et Gélinas, C. (2013). Les diversités au cœur de la recherche interculturelle : vers de nouvelles perspectives. Alterstice, 3(1), 3-8.

White, B. (2014). Quel métier pour l'interculturalisme au Québec?. Dans L. Emongo et B. White (dir.), L'interculturel au Québec. Rencontres historiques et enjeux politiques (p. 21-44). Montréal : Presses de I’Université de Montréal. 